VOL. 1, ISSUE 1, OCT. 2012

\title{
Finger Gesture Based Tablet Interface
}

\author{
V. Shishir Reddy, V. Raghuveer, R. Hithesh, R. Pratesh Kumar Reddy, J. Vamsi Krishna, K. Chandra lohit \\ Department of Electronics and Communication Engineering, M.A.N.I.T, Bhopal, India.
}

\begin{abstract}
Pattern based interaction system provides the user to interact with devices in a more intuitive way. Growth in virtual environments based upon computer systems and development of user interfaces influence the changes in the HumanComputer Interaction (HCI). HCI is a study in which the relationship between humans and computing technology and how computers are designed for easy to use by human, more practical and more intuitive. HCI emphasizes how human interaction with computer technology Pattern recognition based interaction interface, endow with more realistic and immersive interaction compared to the traditional devices. The system enables a physically realistic mode of interaction to the virtual environment. The Pattern recognition system based Interface proposed and implemented in this paper consists of a Detection, recognition and extraction. Comprehensive user acceptability has been considered to exhibit the usefulness and ease of use to the proposed and implemented pattern recognition system. The proposed hand gesture recognition system offers extensions to traditional input devices for interaction with the virtual environments. This type of interaction interface being proposed here can be substantially applied towards many applications like Games. The presented paper considers gadgets as the application domain.
\end{abstract}

Keywords: HCI (Human Computer Interaction), Virtual Interaction, Tablet Interaction.

\section{INTRODUCTION}

Interaction with tablets and mobile phones is done in many different ways these days .In this document we are proposing a new method of interaction with tablets and mobile phones named as virtual touch screen. In the present gadgets and devices, most of the interaction is done with the touch screens, which need direct contact with screen of the device. The virtual touch screen uses highly efficient image processing algorithm (taking processing power as the main concern) using just a camera [it can be already be a part of the device otherwise can be included externally] and a finger with a mark for identification on it. The main inspiration behind this method is advantage of no direct contact with the display screen of the device which increases the durability of devices.

HCI is a discipline of science that focuses on the design, evaluation, and implementation of the interaction of computing systems for human interests, and supports the development of technologies that benefit the environment concerned. Basically there are 4 areas of $\mathrm{HCI}$ and all development is to enhance human interaction with computing systems.

a) Design of an interactive computing system which focuses on how to design a computing system that is easy to use, effective and fun so that is basically a how to find solutions in designing new computing.

b) Implementation of interactive computing system which focuses on how to produce a knowledge of the capabilities of technology and ideas about the development potential that can be utilized so that in this section is how to build applications.

c) Evaluation of interactive computing systems of the discussion focuses on the process whereby data collection systematically used by users or groups of users that can be used in the environment is concerned; this section focuses on the use of data collection and data analysis.

d) Studies of how phenomenon discusses issues such as how the introduction of computer system can affect the work or how the reactions that arise when humans communicate with each other with machines, this section focus on how your study of the development of the human relationship with machines.

In this paper we are focusing mainly on the first defined area i.e. design of interactive computing system.

The development of HCI has developed into virtual interactions (Human Virtual Environment Interaction - HVEI), on HVEI, humans interact with virtual environment using technology developed by HCI, HVEI becoming a trend with the development of HCI research models, HVEI HCI research is how humans interact with virtual environment and communicate with the virtual world and give effect to this virtual environment so that the virtual environment to respond to human interaction. 
VOL. 1, ISSUE 1, OCT. 2012

\section{SET UP}

Virtual touch screen can be used in any devices that require an interaction with a human user. The device which we are considering is a tablet. A normal VGA camera is sufficient to carry on the task of capturing the finger movement. This camera can be inbuilt part of the device or can be interfaced to the device externally. The camera is positioned at the top center of the device (tablet) such that the whole screen of the tablet is in camera's field of view. An orange colour round mark of known size is put on the front side of user's finger, which is meant for operating the tablet. The reason for choosing the round shape is its simplicity and uniqueness to detect. Orange colour is chosen because it stands unique and easy to differentiate from surroundings in most of the situations.

\section{WORKING}

The finger with the mark on it is moved superficially on the surface of the tablet maintaining a distance from the screen [without touching]. The camera captures the video frames of the finger movement. The video frames are processed to extract the orange round mark and then the position of the finger is traced continuously with reference to a previously initialized position. The basic principle employed is when the finger moves from one position to another then the position and also size of the orange circle will change depending on its vectorial position from camera. These areas and positions are compared to the area and position of the initial position and the finger is tracked continuously.

The procedure involves following:

A. Initializing the starting position

B. Extracting the round mark and its parameters from the video frames

C. Estimating the position w.r.t initial position

D. Detecting navigation, click and scroll on the screen.

\section{A. Initialising the starting position}

The position of the finger at any time is traced with respect to an initial position (labeled $\mathbf{O}$ ). The initial position is always fixed at a standard position on the device. When the device is booted (or unlocked) the finger (with round mark facing the camera) is captured by the camera. Then the area and centroid of the initial position's mark is calculated. The coordinates of the standard initial position are already known. The initialization is done just to calculate the area and centroid of the round mark (and thus change the scaling factors) as this will enable the user to use round mark of any size. Thus the user needs the initialization only when user changes the mark or when user needs a recalibration (for accuracy).

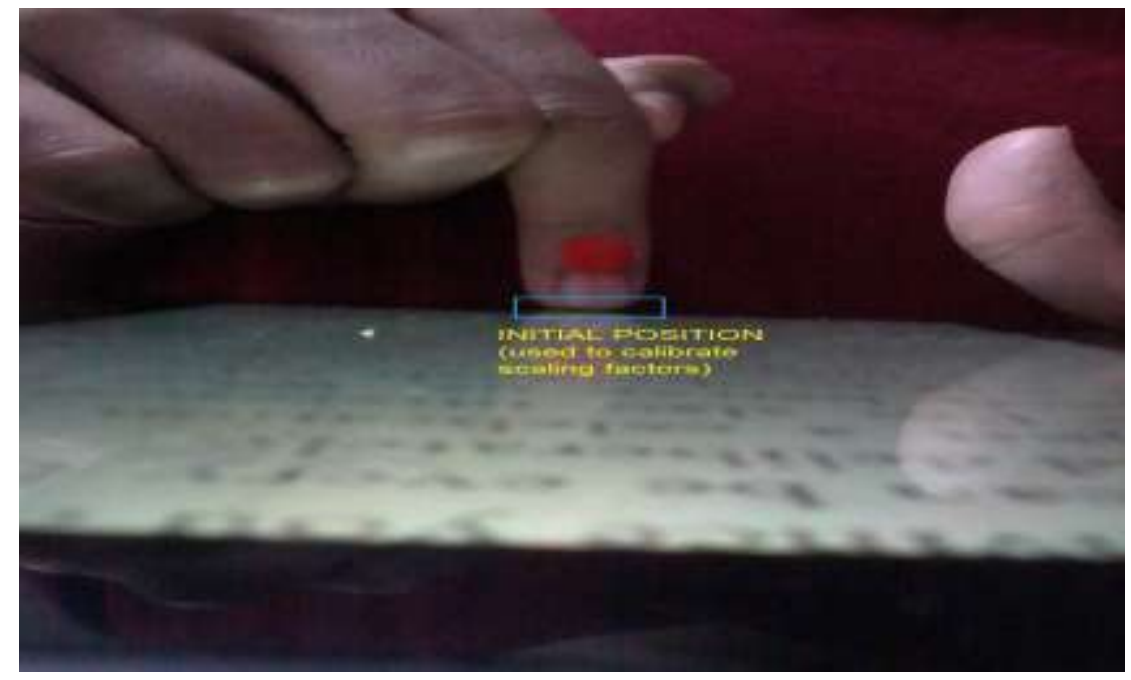

Initialization position 
VOL. 1, ISSUE 1, OCT. 2012

\section{B. Tracking the position of the finger}

After the initialization is over the camera will be continuously capturing the finger movements. Area and centroid of the mark are extracted using the object extraction and tracking process. This whole determination is done taking the initial position, $\mathbf{O}$ as the reference point

At any time t,

\section{AreaDiff $(t)=$ Area of orange mark at (present position - initial position)}

This AreaDiff(t) when multiplied by a pre-determined Y-scaling factor which gives us the $\mathrm{Y}$ coordinate of centroid of orange circle on the finger.

\section{Centdiff $(t)=$ Distance between the (present position- initial position) of the centroid.}

When this Cent.diff(t) is multiplied by a pre-determined X-scaling factor which will provide us with X coordinate of the finger .At any point of time the present position of centroid can be imagined as the projection of the centroid on an imaginary axis passing through $\mathbf{O}$ as shown in fig $(\mathrm{xx})$.

\section{Scaling factors}

There are two pre-determined scaling factors in X and Y directions. These scaling factors are fixed for a particular device (tablet) and round mark of particular area. The scaling factors (Ys and Xs) are constant coefficients.

Ys= vertical dimension of the tablet/(No. of orange round mark pixels in initial position- Total no. of pixels)

$\mathrm{Xs}=$ Horizontal dimension of the tablet/ (No. of pixels between the centroid positions when the finger is placed on the far two corners of the tablet)

The Ys and Xs are determined once for a particular tablet (as they depend on its dimensions). But if a round mark of different area is used then it just changes the Y scaling factor due to change in "no. of orange round mark pixels in initial position".

\section{Detecting the clicks, scrolls and swapping on the surface of the tablet}

Operating the tablet by the user requires clicks and scrolls. To determine them, the Z-dimension coordinates are used. As a rule the user is advised to operate the device by placing the finger at certain minimum height (Hmin) from it. And also the user should not move the finger in either $\mathrm{X}$ or $\mathrm{Y}$ dimensions if he/she desires to click. At any time $\mathrm{t}$, a parameter $\mathrm{H}(\mathrm{t})$ is extracted from the image sequence.

$H(t)=$ No. of pixels between the centroid of the round mark and tablet's surface pixels in the image

If at any point of time this $\mathrm{H}(\mathrm{t})$ becomes less than $\mathrm{H}_{\min }$ for just a minimum time $\mathbf{T}_{\min }$ (say few seconds or a fraction of second) then the system considers it as click at that particular location. But if the $\mathrm{H}(\mathrm{t})$ remains less than $\mathrm{H}_{\min }$ for more than this $\mathrm{T}_{\min }$ then it is no more considered as the click. If the user moves his finger at a height less than $\mathrm{H}_{\min }$ then it is taken by the system as scroll or swapping. Thus $\mathrm{T}_{\min }$ is the threshold time period which decides whether it is a click or scroll (swapping). To scroll vertically the finger is moved in Y direction and to scroll horizontally it's moved in X-direction (but on one condition that $\mathrm{H}(\mathrm{t})<\mathrm{H}$ min).

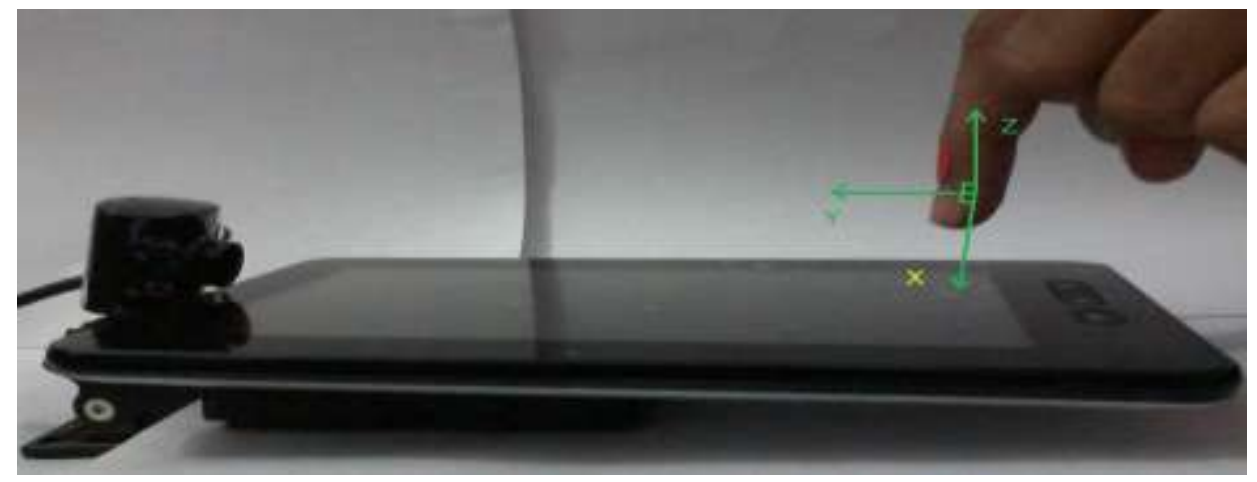


VOL. 1, ISSUE 1, OCT. 2012

\section{ALGORITHM}

- $\quad$ Assume that Xs and Ys scaling factor are known.

- The initial position and parameter are calculated by placing the finger at a standard position.

- $\quad$ The camera captures the images of finger. The round mark on finger is extracted.

- The image with round mark is tuned to binary such that only the circular position is obtained in white region.

- The no. of pixels occupied by the round mark i.e. area is extracted along with its centroid.

- Area Diff(t) is found, multiplied by Ys to give Y coordinate. Cent. Diff(t) is found, multiplied by Xs to give X coordinate.

- If the user doesn't move the finger in either X or Y directions then it have checked if movement is in Z-direction.

- If there is movement in Z-direction and $\mathrm{H}(\mathrm{t})$ is less than $\mathrm{H}_{\min }$ and time period is less than $\mathrm{T}_{\min }$ then it is considered as click in that location.

- If there is movement in Z-direction and $\mathrm{H}(\mathrm{t})$ is less than $\mathrm{H}_{\min }$ but time period is more than $\mathrm{T}_{\min }$ then it is considers as scroll in $\mathrm{Y}$ direction and swap in $\mathrm{X}$ direction.

- The efficiency can be further improved by introducing another parameter frame grab interval. It is the difference in time period between two consecutive video frames which are actually processed by the system.

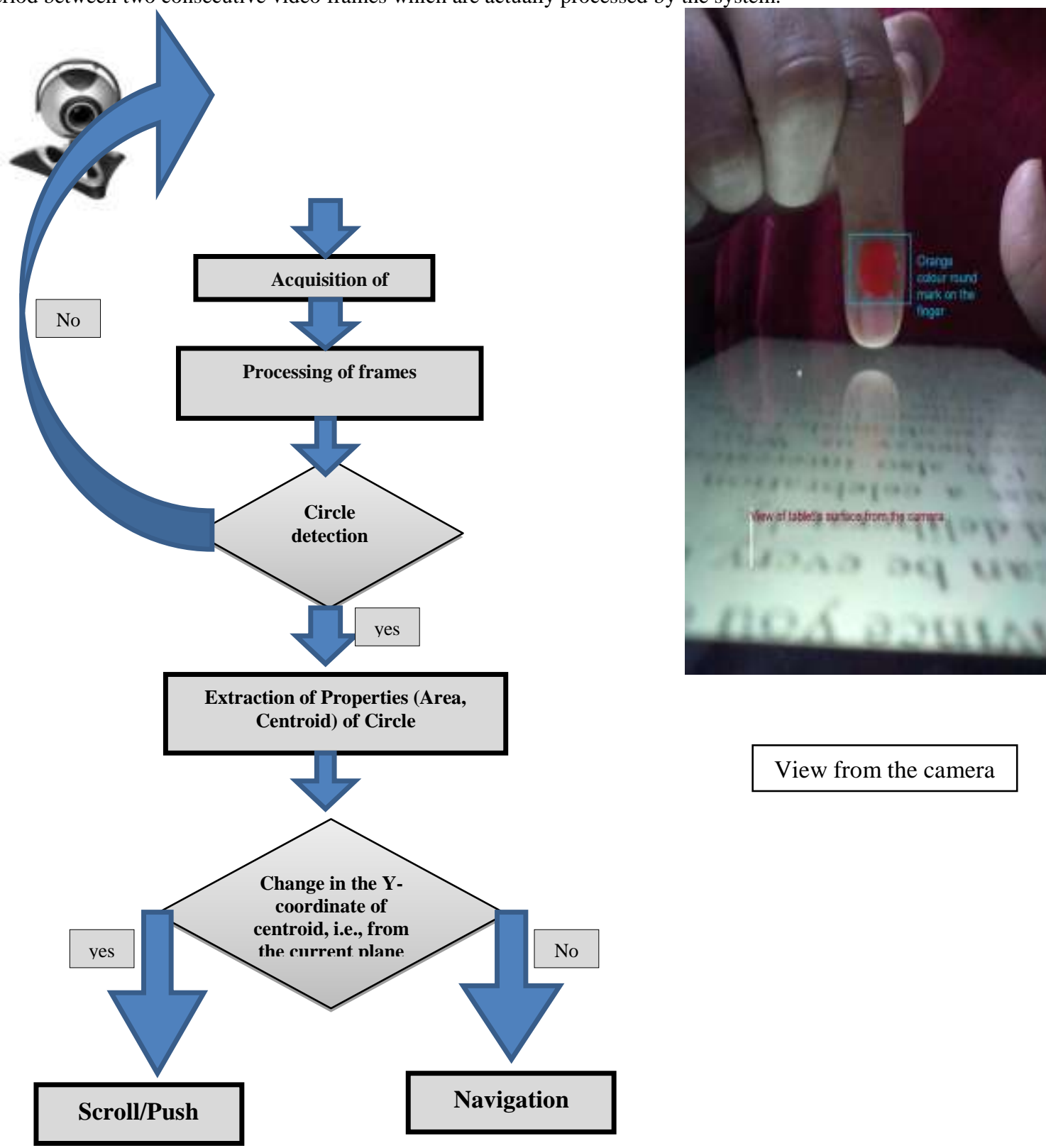


VOL. 1, ISSUE 1, OCT. 2012

\section{RESULT ANALYSIS}

The image processing techniques used in the application for interactions with virtual touch screen have been implemented in MATLAB environment. The web cam used in the experimental setup captures image sequences at the resolution of $640 \times 480$. Practical experiments show that our application is implemented well in environments with little noises (i.e., existence of objects whose color is similar) and with the balanced lightning condition.
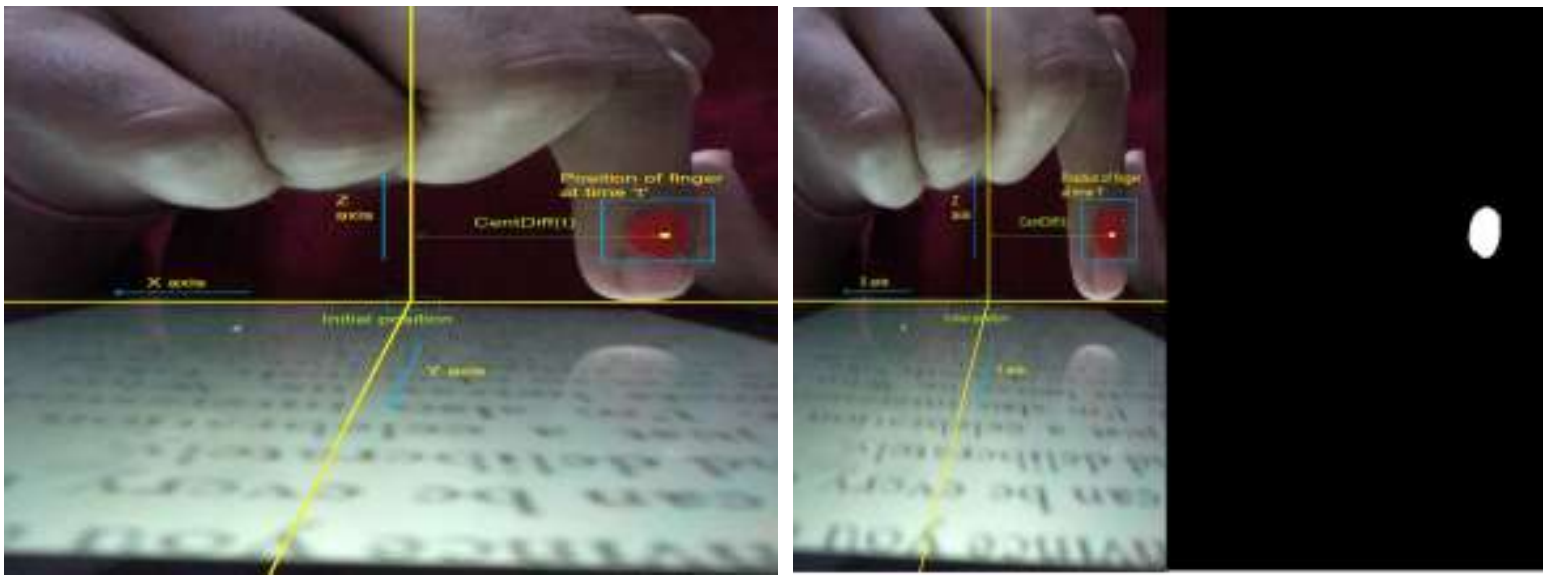

Extraction of the orange mark after processing
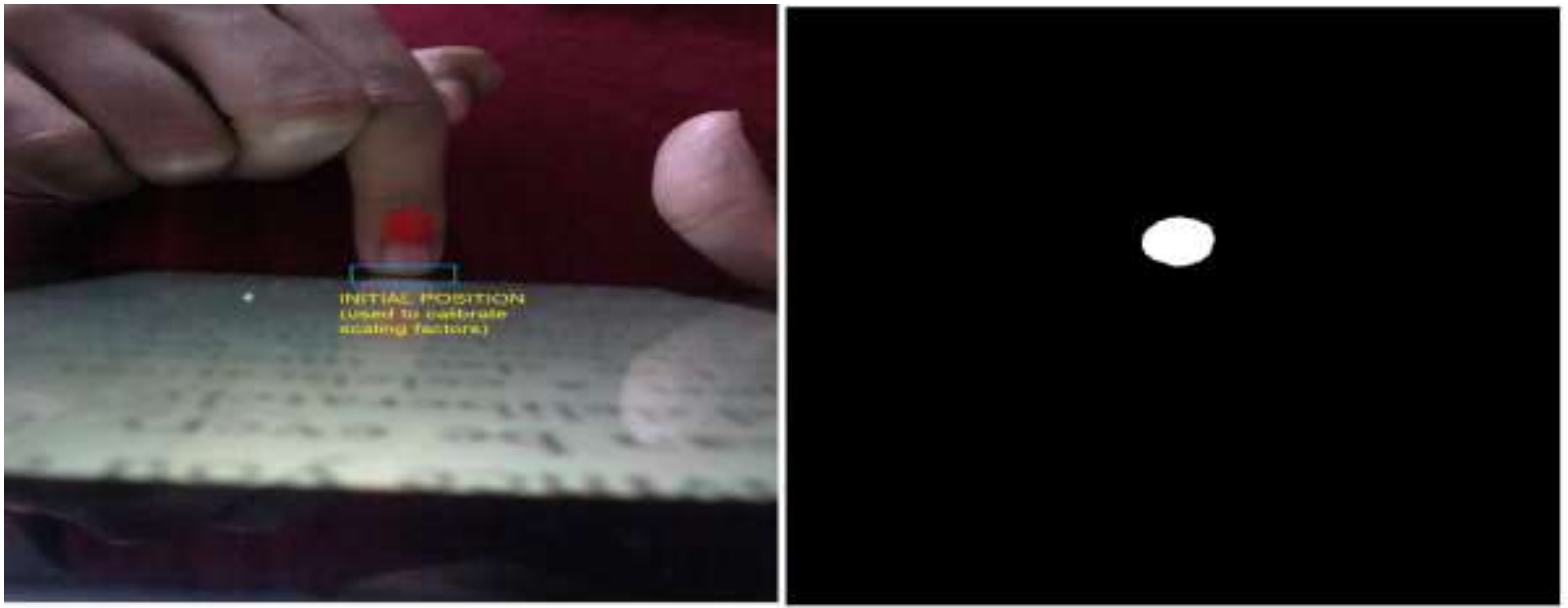

Extraction of the orange mark after processing (initial position)

\section{Conclusion}

The furtherance's in the field of Human-Computer Interaction is majorly been in novel ways of interaction. Hence instead of designing regular interfaces, research branches are exploring new ways in interacting with the devices. Many of input providing technologies require physical touch and also there are other variations that provide input to the application without using physical touch. The advantage of the usage of hand gesture based input mode is that this method provides the user a new ways of interacting with the application without using the traditional input devices like keyboard or mouse. The application of virtual touchscreen controlling through hand movement proposed and implemented in the present paper provides a suitable efficient and user friendly interface between human and computer for interaction. The user also can have an interaction with the applications through an interface that provide gesture as an input according to interested function that can be developed within the applications.

\section{REFERENCES}

[1]. Rafael C.Gonzalez and Richard E. Woods, "Digital Image Processing". 